\title{
Novel DCPD-modified polyester containing epoxy groups: thermal, viscoelastic, and mechanical properties of their wood flour filled copolymers
}

\author{
Marta Worzakowska
}

Received: 30 May 2011/Revised: 11 July 2011/Accepted: 16 July 2011/

Published online: 27 July 2011

(C) The Author(s) 2011. This article is published with open access at Springerlink.com

\begin{abstract}
In this article, the synthesis of novel DCPD-modified polyesters containing epoxy groups and their possible utilization as polar matrices for wood flour filled copolymers have been studied. The novel DCPD-modified polyester containing epoxy groups was prepared during three-step process. First, the addition reaction of maleic acid to DCPD norbonenyl double bond has been performed. Then, the polyesterification of acidic ester of DCPD, cyclohex-4-ene dicarboxylic anhydride, and ethylene glycol (ethane-1,2-diol, EG) or neopentyl glycol (2,2dimethyl-1,3-propanediol, NP) in the presence of catalyst was carried out using melt condensation technique. Finally, the oxidation process of prepared DCPD-modified polyesters led to obtain DCPD-modified polyesters containing epoxy groups. The structural characterization of prepared DCPD-modified polyesters containing epoxy functionality has been carried out using FTIR and HNMR spectroscopic methods. The thermal, viscoelastic, and mechanical properties of their unfilled and wood flour filled copolymers have been studied by DSC, DMA, TGA analyses, three-point bending test, and Brinell's hardness. The performed investigations indicated that novel DCPD-modified polyesters containing epoxy groups can be successfully applied as polar matrices for preparation wood flour filled copolymers due to their "compatibility" with hydrophilic wood flour surface which resulted with stronger interactions between wood and polymer surface and thus producing copolymers which were characterized by improved thermo-mechanical properties compared to unfilled copolymers.
\end{abstract}

Keywords Dicyclopentadiene - Polyesters - Wood flour - Thermal properties · Mechanical properties

\footnotetext{
M. Worzakowska $(\bowtie)$

Department of Polymer Chemistry, Faculty of Chemistry, Maria Curie-Sklodowska University, Gliniana 33 Street, 20-614 Lublin, Poland e-mail: marta.worzakowska@poczta.umcs.lublin.pl
} 


\section{Introduction}

The properties of polyesters and their cured products largely depend on the nature of chemical structure of the starting resins. The incorporation of various types of moieties into the polyester backbone allows tailoring and preparation of various resins for different applications [1]. One of the compounds that can be widely applied as a comonomer for polyester synthesis is dicyclopentadiene (DCPD) due to their availability, low cost, and reactivity [2, 3].

DCPD is the Diels-Alder reaction dimmer of cyclopentadiene (CPD), their thermodynamically stable form at room temperature. This reagent is mainly obtained as a by-product in the olefin cracking process or during the synthesis of ethylene [3]. The difference in the reactivity between the norbornene and cyclopentene double bonds in DCPD allows preparing their derivatives which can be successfully used as an intermediate for various syntheses [4]. The addition reaction of various compounds, e.g., alcohols, phenols, glycols, mercaptans, di/carboxylic acids, or water preferentially at the norbornenyl double bond of DCPD without involvement of the markedly less reactive cyclopentene double bonds resulting in production of their ester derivatives and secondary alcohols [5, 6].

The principal methods of DCPD utilization for polyester modification include two methods depending on temperature. In the temperature range of $120-140{ }^{\circ} \mathrm{C}$, the nucleophilic addition of carboxylic or hydroxylic groups from dicarboxylic acids or glycols to more reactive double bond of DCPD at the norbornenyl ring is mainly expected and thus led to obtain ester or ether derivatives of DCPD. However, at temperatures above $150{ }^{\circ} \mathrm{C}$, the de-dimerization of DCPD to CPD and the addition of CPD to unsaturated double bonds in polyester chains results in obtaining cycloalkene structure in their backbone [7]. The ability to incorporate a large amount of bulky and highly aliphatic DCPD moiety into polyester structure allow preparing less expensive polyesters which are characterized by reduced shrinkage during cure [3]. In addition, DCPD and their based products can be easily chemically modified using hydrogen peroxide or peracids and thus forming their epoxy derivatives [8]. The resulting epoxides display high reactivity attributed to the high ring strain present in the epoxycyclopentenyl ring system. The chemically modified DCPD-based polymers possess excellent rigidity, mechanical strength, high thermal stability, electrical properties after curing, high dimensional stability, and highly resistant to ultraviolet light [6]. They are attractive materials for a wide industrial application such as paints, coatings, matrices, packing materials for printed circuit boards, outdoor applications, etc. [9-15].

In this article, the synthesis of novel DCPD-modified polyesters containing epoxy groups and their possible utilization as polar matrices for wood flour filled copolymers have been presented. The structural characterization of prepared DCPDmodified polyesters containing epoxy groups have been carried out using FTIR and HNMR spectroscopic methods. The thermal, viscoelastic, and mechanical properties of their unfilled and wood flour filled copolymers have been studied by DSC, DMA, TGA analyses, three-point bending test, and Brinell's hardness. 


\section{Experimental part}

\section{Materials}

DCPD, maleic acid (MA), ethylene glycol (ethane-1,2-diol, EG), neopentyl glycol (2,2-dimethyl-1,3-propanediol, NP), cyclohex-4-ene-1,2-dicarboxylic anhydride (THPA), hexahydrophthalic anhydride (HHPA) and $40 \%$ peracetic acid were obtained from Merck-Schuchardt, Germany. Butylstannoic acid (catalyst) was from Arkema Inc., USA. Benzoyl peroxide (BPO) was supplied by Fluka, Switzerland. Styrene (ST), hydoquinon, and xylene were delivered by POCh, Poland.

\section{Synthesis of DCPD-modified polyesters containing epoxy functionality}

DCPD (1.07 mol), MA (1 mol), and hydroquinone $(0.035 \mathrm{wt} \%)$ were placed into a $500 \mathrm{~mL}$ three-necked flask equipped with a mechanical stirrer, thermometer, and condenser. The mixture was heated to $135^{\circ} \mathrm{C}$, and stirred rigorously for a further $2 \mathrm{~h}$ maintained at this temperature, under reflux. The drop of an acid value to $226 \mathrm{mgKOH} / \mathrm{g}$ was an indicator of the process completion. In this stage, acidic ester of DCPD has been obtained (Step I, Scheme 1).

Then, after cooling, to the acidic ester (Stage I), ethylene glycol or neopentyl glycol $(2.15 \mathrm{~mol})$, cyclohex-4-ene-1,2-dicarboxylic anhydride $(1.5 \mathrm{~mol})$, and catalyst $(0.52 \mathrm{~g})$ were added. The reaction mixture was heated up to $150{ }^{\circ} \mathrm{C}$ for $1 \mathrm{~h}$,

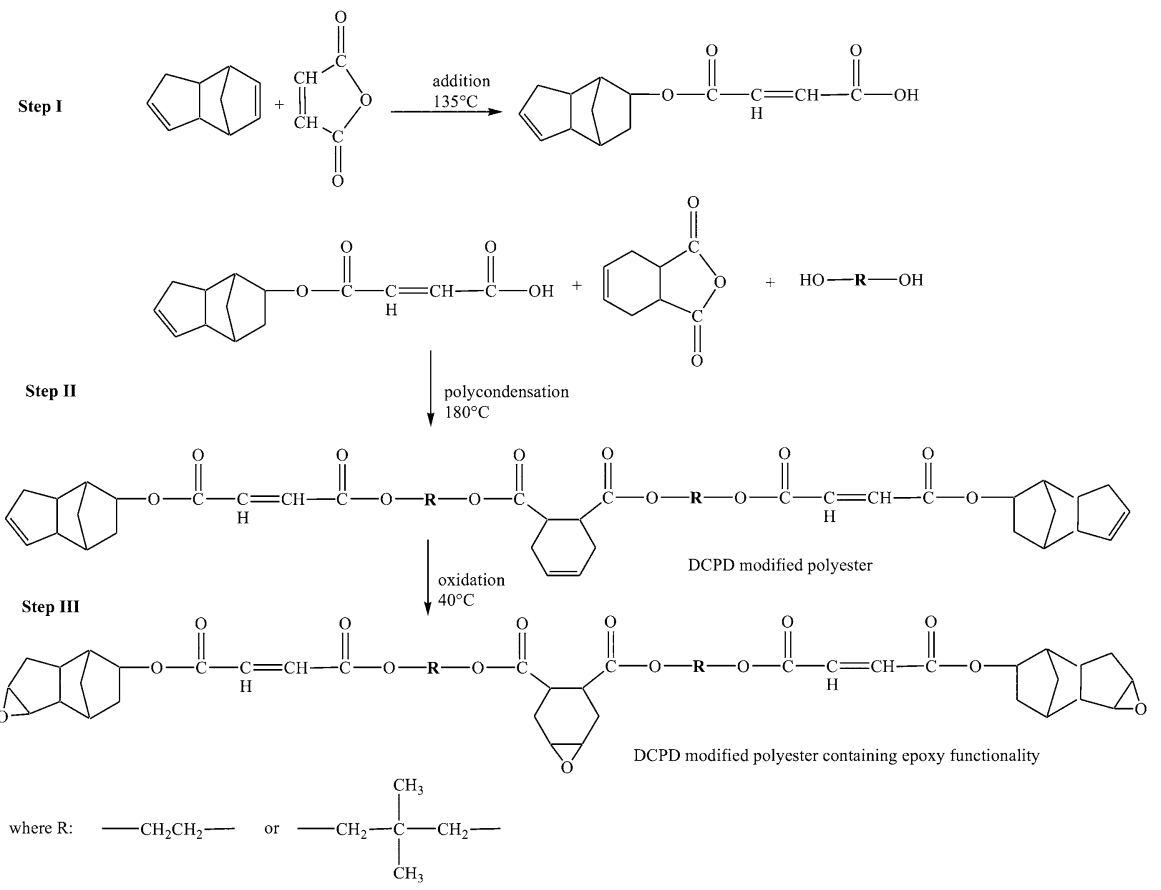

Scheme 1 The scheme of synthesis of novel DCPD-modified polyester containing epoxy groups 
and then heated to $180{ }^{\circ} \mathrm{C}$ and maintained at this temperature until the drop of an acid value below $3 \mathrm{mgKOH} / \mathrm{g}$. Acid value $(\mathrm{mgKOH} / \mathrm{g})$ defined as a number of $\mathrm{mg}$ $\mathrm{KOH}$ required for titration of $1 \mathrm{~g}$ of a sample was determined by titration method. During the reaction, water was removed continuously from the system by azotropic distillation (xylene). After that, xylene was distilled under reduced pressure, and the obtained product was identified, (Step II, Scheme 1).

The preparation of novel DCPD-modified polyesters containing epoxy groups was performed in three-necked flask equipped with a glass stirrer, thermometer, and an addition funnel. The DCPD-modified polyester (100 g) was placed in a threenecked flask dissolved in methylene chloride $(200 \mathrm{~mL})$ and heated up to $40{ }^{\circ} \mathrm{C}$. Than, peracetic acid was slowly added dropwise under vigorous stirring over $1 \mathrm{~h}$. The reaction mixture was then stirred rigorously for a further $3 \mathrm{~h}$ maintained at the reaction temperature. After cooling, the reactive mixture was washed with an aqueous solution of sodium carbonate to neutralization. Organic phase was thoroughly washed two times with distilled water, dried over anhydrous $\mathrm{MgSO}_{4}$, and distilled under vacuum to remove solvent. Epoxy value $(\mathrm{mol} / 100 \mathrm{~g})$ of prepared novel polyesters determined by dioxane $/ \mathrm{HCl}$ titration method was 0.35 and 0.33 , respectively. The theoretical structure of novel DCPD-modified polyesters containing epoxy groups was presented in Scheme 1, Step III.

Characterization of novel DCPD-modified polyesters containing epoxy functionality

Fourier transform infrared (FTIR) spectra were obtained using a Perkin-Elmer 1725 $\mathrm{X}$ FTIR spectrophotometer in the $400-4000 \mathrm{~cm}^{-1}$ wavenumber range using $\mathrm{KBr}$ pellets.

Proton nuclear magnetic resonance $\left({ }^{1} \mathrm{H}\right.$ NMR) spectra were recorded on an NMR Brucker-Avance $300 \mathrm{MSL}$ (Germany) spectrometer at $300 \mathrm{MHz}$ with deuterated chloroform $\left(\mathrm{CDCl}_{3}\right)$ as the solvent. ${ }^{1} \mathrm{H}$ NMR chemical shifts in parts per million (ppm) were reported downfield from $0.00 \mathrm{ppm}$ using tetramethylsilane (TMS) as an internal reference.

\section{Preparation of copolymers}

Wood flour filled copolymers were prepared by mixing novel DCPD-modified polyesters containing epoxy groups, styrene (20 wt $\%)$, BPO (1.0 wt $\%$ ), a stoichiometric ratio of an HHPA, and different amounts of 1, 5, 10, 20, 40, and $60 \mathrm{wt} \%$ of unmodified wood flour filler dried in a vacuum oven at $70{ }^{\circ} \mathrm{C}$ to remove moisture before making copolymers. To compare the studied properties, samples of unfilled copolymers as well as filled with alkali-treated $(10 \% \mathrm{NaOH}$ for $2 \mathrm{~h}$ ) wood flour filler $(20 \mathrm{wt} \%)$ were prepared for the investigations. All components were mixed together, heated to $40{ }^{\circ} \mathrm{C}$, and stirred for about $1 \mathrm{~h}$ with the assistance of mechanical stirrer to obtain uniform dispersion. All the compositions (pastes) were placed in a glass mold under vacuum at $50{ }^{\circ} \mathrm{C}$ for degassing, cured at $80{ }^{\circ} \mathrm{C}$ for $6 \mathrm{~h}$, and then post-cured in an oven over $6 \mathrm{~h}$ at $140-150{ }^{\circ} \mathrm{C}$. 
Characterization of copolymers

Thermogravimetric analysis (TGA) was carried out on a MOM 3427 derivatograph Paulik and Erdey (Hungary) at a heating rate of $10{ }^{\circ} \mathrm{C} / \mathrm{min}$ in air in the temperature range of $20-1000{ }^{\circ} \mathrm{C}$ with the sample weight of $10 \mathrm{mg} . \alpha-\mathrm{Al}_{2} \mathrm{O}_{3}$ was used as a reference. The initial decomposition temperature (IDT), the temperature of the maximum rate of weight loss $\left(T_{\max }\right)$, and the final decomposition temperature (FDT) were determined.

DMA measurements were performed using Dynamic Mechanical Analyzer (DMA) Q 800 TA Instruments (USA). Tests were conducted with a double Cantilever device with a support span of $35 \mathrm{~mm}$, calibrated according to the producer's recommendation. Temperature scanning from room temperature until the sample become too soft to be tested with a constant heating rate of $4{ }^{\circ} \mathrm{C} / \mathrm{min}$ at an oscillation frequency of $10 \mathrm{~Hz}$ was performed. Rectangular samples of $10-\mathrm{mm}$ wide and 4-mm thick were used. Dynamic mechanical properties were estimated from the changes of storage modulus $\left(E^{\prime}\right)$, mechanical loss $\left(E^{\prime \prime}\right)$ as well as from the changes of $\tan \delta$ at constant frequency depending on temperature. Glass transition temperature ( $\alpha$-relaxation) was taken as the maximum of the $\tan \delta$ versus temperature curves.

Flexural properties were measured in a three-point bending test using a Zwick/ Roell Strength Machine (model Z010, Germany). The specimen dimensions were $10-\mathrm{mm}$ wide and 4-mm thick. The measurements were carried out at room temperature with a crosshead speed of $2 \mathrm{~mm} / \mathrm{min}$ using a support span of $64 \mathrm{~mm}$. At least five specimens were tested for each sample to evaluate flexural properties. The flexural modulus at bending $\left(E_{\bmod }\right)$, deflection at maximum force $\left(\varepsilon-F_{\max }\right)$, and flexural stress $(\sigma)$ were qualified.

Hardness according to Brinell was determined by means of a hardness tester HPK and calculated based on following equation: $\mathrm{HK}[\mathrm{MPa}]=F_{1} * 0.098066$, where $F_{1}$ is a strength of pressure under definite load.

\section{Results and discussion}

Characterization of novel DCPD-modified polyesters containing epoxy groups

The novel DCPD-modified polyesters containing epoxy groups were characterized by FTIR and ${ }^{1} \mathrm{H}$ NMR. The presence of absorption peaks at $781-835 \mathrm{~cm}^{-1}$ characteristic for oxirane ring vibration groups at cycloaliphatic rings are clearly observed. In addition, the presence of absorption signals at $985-990 \mathrm{~cm}^{-1}$ $\left(-\mathrm{CH}=\mathrm{CH}\right.$ wagging of maleic anhydride) and $1646-1648 \mathrm{~cm}^{-1}(\mathrm{C}=\mathrm{C}$ stretching vibration for carbon-carbon double bonds of maleic anhydride) was indicated, which confirmed the presence of carbon-carbon double bonds (cis-trans units) in the structure of DCPD-modified polyesters containing epoxy groups, Fig. 1.

The exemplary ${ }^{1} \mathrm{H}$ NMR spectrum of DCPD-modified polyester containing epoxy groups was shown in Fig. 2. The corresponding proton signals at 6.30-6.35 and 6.90-6.95 ppm were due to carbon-carbon double bonds (cis-trans units of 


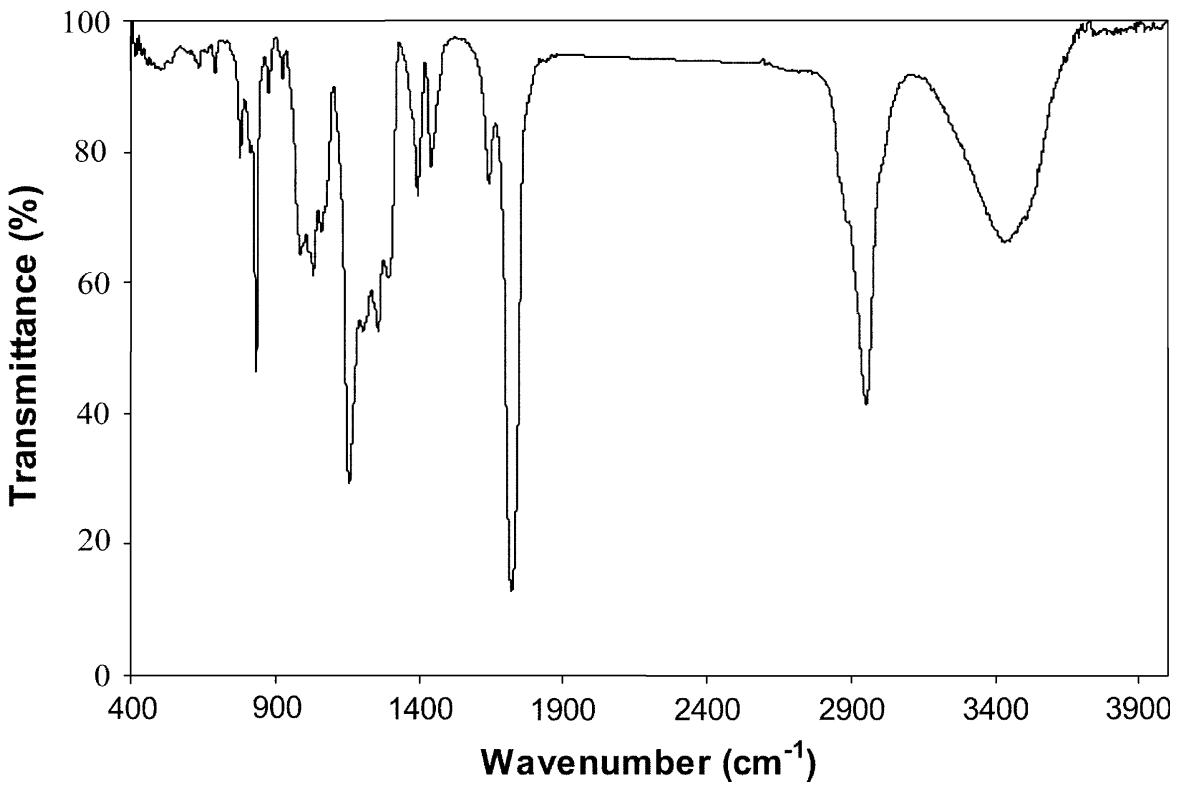

Fig. 1 FTIR spectra of novel DCPD-modified polyester containing epoxy groups

maleic anhydride). The chemical shifts of the protons in surroundings of epoxy groups $\delta=3.2-3.5$ were clearly observed.

Characterization of copolymers

The data obtained from DMA curves for prepared untreated, alkali-treated wood flour filled copolymers as well as unfilled copolymers are presented in Tables 1 and 2. In addition, the variation of the loss modulus $\left(E^{\prime \prime}\right)$ and the $\tan \delta$ of unfilled copolymers and different amounts of wood flour filled copolymers (1-60 wt \%) and alkali-treated wood flour filled copolymers are shown in Fig. 3. Based on visible results, the values of storage modulus $\left(E_{20}^{\prime}{ }^{\circ} \mathrm{C}\right)$ increased with the increase of wood flour filler content (1-40 wt\%) in copolymers compared to unfilled copolymers, Tables 1 and 2.

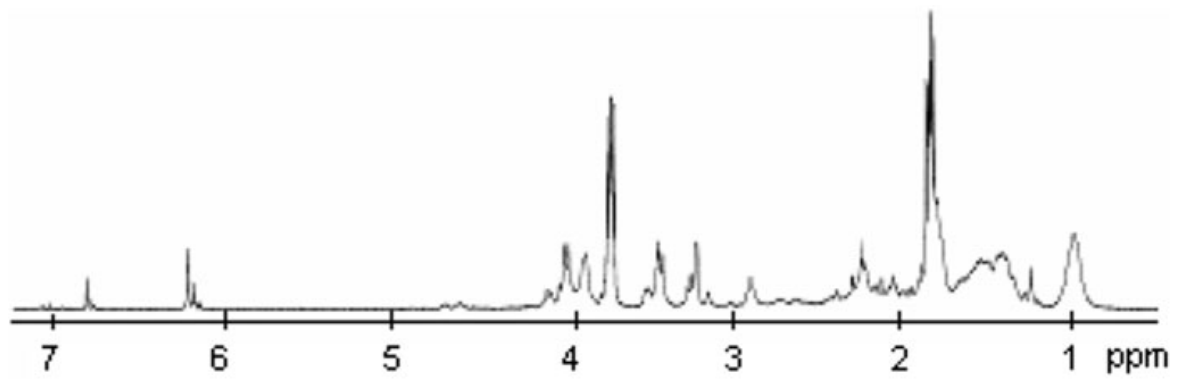

Fig. $2{ }^{1} \mathrm{H}$ NMR spectra of novel DCPD-modified polyester containing epoxy groups 


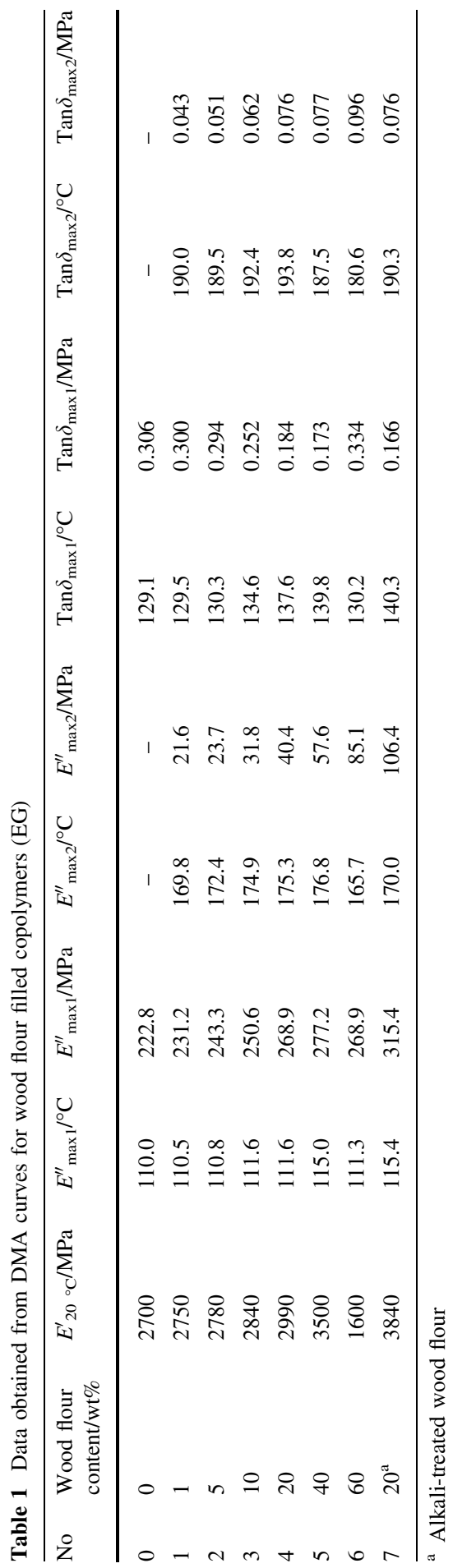




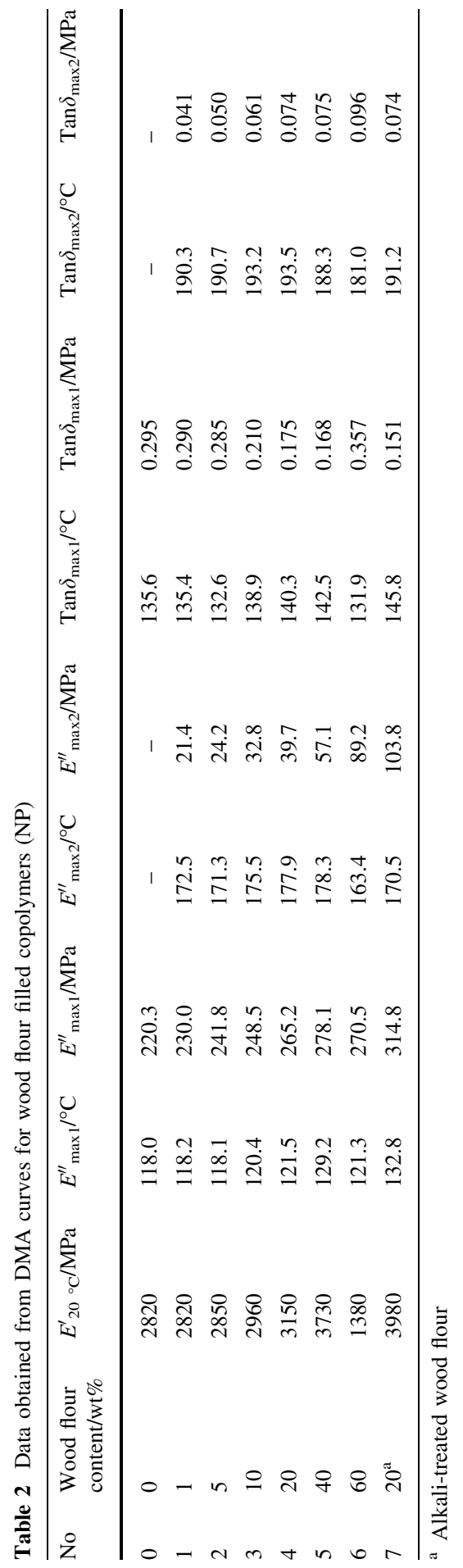



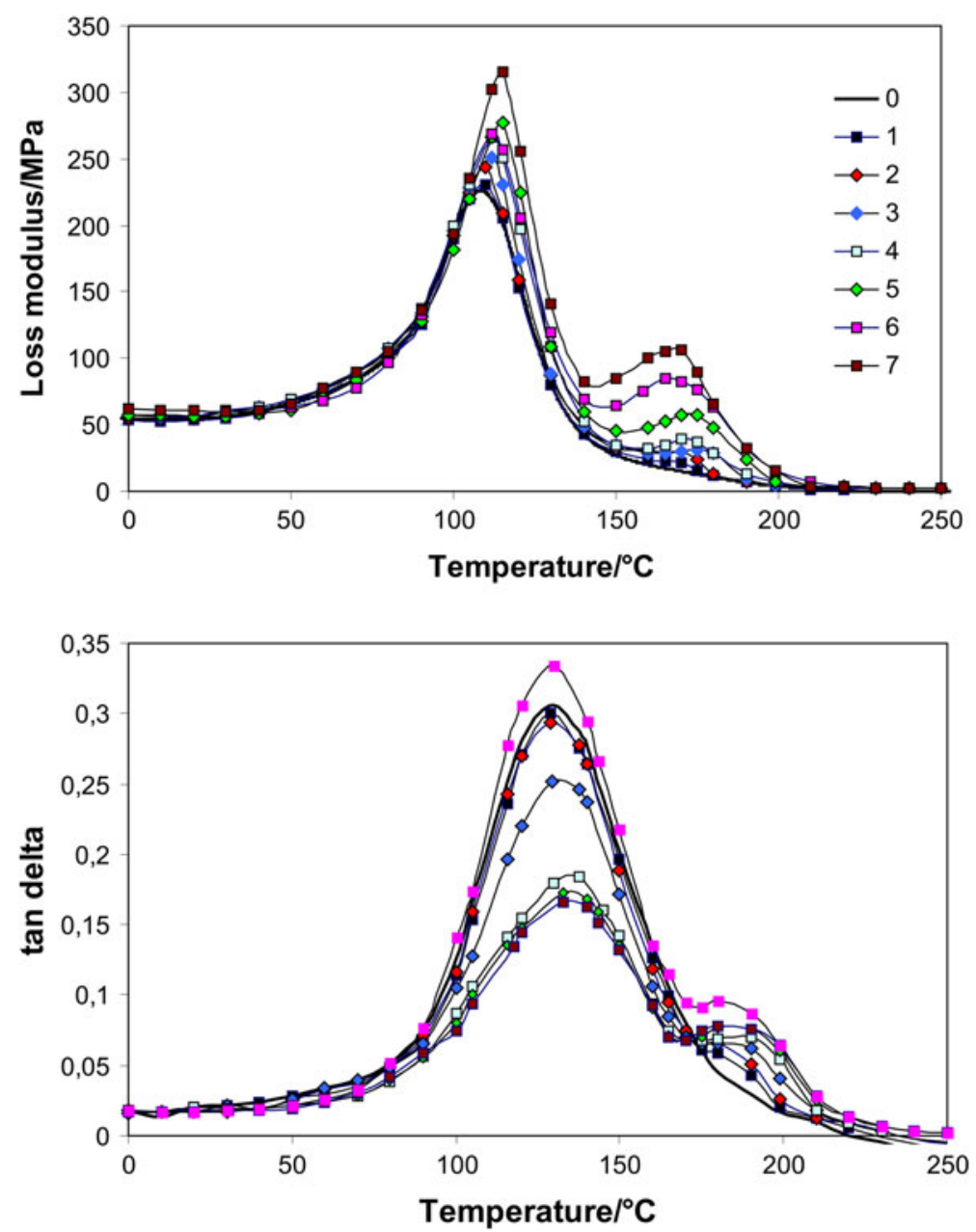

Fig. 3 The variation of the loss modulus $\left(E^{\prime \prime}\right)$ and the $\tan \delta$ of the $(0)$ unfilled resin, (1) $1 \% \mathrm{WF}$, (2) 5\% $\mathrm{WF}$, (3) $10 \% \mathrm{WF}$, (4) $20 \% \mathrm{WF}$, (5) $40 \% \mathrm{WF}$, (6) $60 \% \mathrm{WF}$, and (7) $20 \%$ alkali-treated WF (WF wood flour)

However, for copolymers containing $60 \mathrm{wt} \%$ of wood flour filler significant fall of $E^{\prime}{ }_{20}^{\circ} \mathrm{C}$ Values were observed. It indicated that the use of wood flour filler at $1-40 \mathrm{wt} \%$ content acted as a reinforcement which allowed obtaining stiffer materials probably due to the greater stress transfer at the interface by the wood flour particles. On the contrary, the tendency to agglomeration of wood flour particles used at higher content resulted in dimensional instability and thus poor mechanical properties of the copolymers. Moreover, the $E_{20}^{\prime}{ }^{\circ} \mathrm{C}$ values of alkali-treated wood flour filled copolymers were higher than that of untreated wood flour filled copolymers. It could be connected with the improvement of interfacial adhesion between wood flour particles and novel DCPD-modified polyesters containing epoxy groups (matrices) due to the formation of additional hydrophilic groups (hydroxyl groups) on the 
particles surface as well as due to the formation more rough surface of the filler [16]. The increase in hydrophilic nature of wood flour particles makes the filler more compatible with more polar matrices. Also, the rough surface improves the mechanical interlocking of the polyester molecules with the filler as well as the adhesion between filler and matrix. As a result of those, the interfacial interaction between those two components increases due to the formation of hydrogen bonding with hydroxyl groups of the filler and the hydroxyl, carboxyl, ester, or epoxy groups present in the novel DCPD-modified polyesters containing epoxy groups.

The one loss modulus peak $\left(E^{\prime \prime}{ }_{\max 1}\right)$ as well as $\tan \delta_{\max 1}$ for unfilled copolymers was indicated, respectively. This peak was attributed to the mobility of the resin molecules [17]. In the wood flour filled copolymers, the position of $E^{\prime \prime}{ }_{\max 1}$ and $\tan \delta_{\max 1}$ was almost independent of the wood flour content. However, $\tan \delta_{\max 1}$ height was drastically decreased with increasing filler content in copolymers compared to unfilled copolymers. The $\tan \delta_{\max 1}$ height of the unfilled copolymers was due to the deformation of the resin molecules between the cross links on application of stress. However, the incorporation of the reinforcing filler significantly restricted the mobility of the polyesters molecules in prepared copolymers and thus raised the $E_{20}^{\prime}{ }^{\circ} \mathrm{C}$ values and reduced the $\tan \delta_{\max 1}$ height, which probably indicated on the effective incorporation of the reinforcing filler into polymer matrix leading to formation of a stronger bond at the interface and thus increasing the relative thickness of the bonded interface [18-20].

In addition, at higher temperature, a small hump only for wood flour filled copolymers was observed, Fig. 3. This hump became more prominent and broader with the increase of wood flour filler content in the copolymers and thus probably connected with cellulose component, their glass transition temperature, which was nearly $200{ }^{\circ} \mathrm{C}$ according to literature data $[21,22]$.

The glass transition temperature (described as $\tan \delta_{\max 1}$ ) of the wood flour filled copolymers was found to have imperceptibly shifted to higher temperatures with increase in wood flour loading up to $40 \mathrm{wt} \%$. Also, the increase in $\tan \delta_{\max 1}$ for alkali-treated wood flour filled copolymers was indicated. It may be attributed to better interfacial interactions in alkali-treated filled copolymers, which significantly decreased the polymer chain mobility, Tables 1 and 2 .

Thermal decomposition (IDT) of wood flour filled copolymers started at higher temperatures (about $10-50{ }^{\circ} \mathrm{C}$ higher) than that of neat wood flour filler $\left(240{ }^{\circ} \mathrm{C}\right)$, Tables 3 and 4. Also, the results showed that there was a shift to higher temperatures of the peak that correspond to the main degradation process of the wood flour component $\left(T_{\max 1}\right)$. This observation could indicate on stronger interactions between more polar matrices and wood flour component by creating intermolecular bonding between components and thus the presence of polymer matrices protected the filler delaying its degradation [23].

The thermal degradation pattern of unfilled copolymers exhibited two almost separated degradation steps, with two maximum rate peaks $\left(T_{\max }\right)$ at $375-380$ and $575{ }^{\circ} \mathrm{C}$, which were directly associated with the ester/ether and carbon-carbon bonds breakdown formed in copolymers structure during cure [24]. However, wood flour filler as well as wood flour filled copolymers undergoes a complex degradation scheme due to their composition. As it is generally known, wood flour is composed 
Table 3 Data obtained from TGA analysis for wood filled copolymers (EG)

a Alkali-treated wood flour

Table 4 Data obtained from TGA analysis for wood filled copolymers (NP)

a Alkali-treated wood flour

Table 5 Mechanical properties of wood flour filled copolymers (EG)

a Alkali-treated wood flour

\begin{tabular}{llllll}
\hline $\begin{array}{l}\text { Wood flour } \\
\text { content/wt } \%\end{array}$ & IDT $/{ }^{\circ} \mathrm{C}$ & $T_{\max 0} /{ }^{\circ} \mathrm{C}$ & $T_{\max 1} /{ }^{\circ} \mathrm{C}$ & $T_{\max 2} /{ }^{\circ} \mathrm{C}$ & $\mathrm{FDT} /{ }^{\circ} \mathrm{C}$ \\
\hline 0 & 280 & - & 380 & 575 & 635 \\
1 & 280 & 90 & $335 / 385$ & 575 & 635 \\
5 & 275 & 95 & $335 / 385$ & 570 & 630 \\
10 & 275 & 90 & $350 / 390$ & 560 & 630 \\
20 & 270 & 100 & $345 / 385$ & 560 & 630 \\
40 & 260 & 95 & $345 / 380$ & 550 & 635 \\
60 & 250 & 100 & $330 / 375$ & 550 & 630 \\
$20^{\mathrm{a}}$ & 255 & 110 & $360 / 390$ & 565 & 635 \\
Wood flour & 240 & 90 & 320 & 510 & 600 \\
\hline & & & & & \\
\hline
\end{tabular}

\begin{tabular}{llllll}
\hline $\begin{array}{l}\text { Wood flour } \\
\text { content } / w t \%\end{array}$ & IDT $/{ }^{\circ} \mathrm{C}$ & $T_{\max 0} /{ }^{\circ} \mathrm{C}$ & $T_{\max 1} /{ }^{\circ} \mathrm{C}$ & $T_{\max 2} /{ }^{\circ} \mathrm{C}$ & $\mathrm{FDT} /{ }^{\circ} \mathrm{C}$ \\
\hline 0 & 290 & - & 375 & 575 & 640 \\
1 & 290 & 90 & $330 / 375$ & 570 & 640 \\
5 & 280 & 90 & $335 / 380$ & 570 & 630 \\
10 & 275 & 90 & $355 / 380$ & 570 & 630 \\
20 & 275 & 95 & $345 / 380$ & 565 & 635 \\
40 & 270 & 95 & $345 / 385$ & 560 & 635 \\
60 & 250 & 100 & $335 / 385$ & 550 & 630 \\
20 & 260 & 100 & $350 / 380$ & 560 & 630 \\
Wood flour & 240 & 90 & 320 & 510 & 600 \\
\hline
\end{tabular}

\begin{tabular}{lllll}
\hline $\begin{array}{l}\text { Wood flour } \\
\text { content/wt } \%\end{array}$ & $E_{\text {mod }} / \mathrm{GPa}$ & $\varepsilon-F_{\max } / \%$ & $\sigma / \mathrm{MPa}$ & $\mathrm{HK} / \mathrm{MPa}$ \\
\hline 0 & 2.8 & 2.40 & 78.2 & 127 \\
1 & 2.8 & 2.34 & 75.4 & 129 \\
5 & 2.9 & 2.15 & 77.2 & 131 \\
10 & 3.2 & 2.03 & 80.3 & 136 \\
20 & 3.5 & 1.80 & 85.8 & 143 \\
40 & 3.9 & 1.52 & 92.6 & 164 \\
60 & 1.8 & 0.60 & 15.4 & 90 \\
$20^{\mathrm{a}}$ & 4.0 & 1.22 & 102.1 & 169 \\
\hline
\end{tabular}

mainly by three polymers: cellulose, hemicellulose, and lignin and thus each of those polymers has own characteristic thermal degradation properties [25-27]. Three degradation steps for wood flour filled copolymers were observed, which was directly connected with the presence of the filler, Tables 3 and 4. 
Table 6 Mechanical properties of wood flour filled copolymers (NP)

a Alkali-treated wood flour

\begin{tabular}{lllll}
\hline $\begin{array}{l}\text { Wood flour } \\
\text { content/wt } \%\end{array}$ & $E_{\text {mod }} / \mathrm{GPa}$ & $\varepsilon-F_{\text {max }} / \%$ & $\sigma / \mathrm{MPa}$ & $\mathrm{HK} / \mathrm{MPa}$ \\
\hline 0 & 2.9 & 2.20 & 80.5 & 135 \\
1 & 2.8 & 2.28 & 82.2 & 138 \\
5 & 2.9 & 2.25 & 80.5 & 139 \\
10 & 3.3 & 2.00 & 85.2 & 145 \\
20 & 3.4 & 1.60 & 90.5 & 156 \\
40 & 4.1 & 1.45 & 94.8 & 168 \\
60 & 1.5 & 0.35 & 18.2 & 86 \\
$20^{\mathrm{a}}$ & 4.1 & 1.18 & 106.0 & 174 \\
\hline
\end{tabular}

Also, the mechanical studies confirmed earlier observations, Tables 5 and 6 . Wood flour filled copolymers containing 1-40 wt\% of the filler were characterized by higher flexural modulus at bending $\left(E_{\bmod }\right)$, flexural stress $(\sigma)$ as well as Brinell's hardness (HK) values comparing to unfilled copolymers. The increase in mechanical properties was more distinctive at higher wood flour content copolymers. It confirmed the reinforcement effect of wood flour due to the compatibility between hydrophilic surface components: filler-polyester and thus produced more stiff materials. However, the tendency to agglomeration of wood flour particles used at higher content $(60 \mathrm{wt} \%)$ probably resulted in dimensional instability and thus decreased $E_{\text {mod }}, \sigma$, and $\mathrm{HK}$ values resulting in poor mechanical properties of prepared copolymers.

\section{Conclusion}

The synthesis of novel DCPD-modified polyesters containing epoxy groups and their possible utilization as polar matrices for wood flour filled copolymers have been presented. The performed studies indicated that novel DCPD-modified polyesters containing epoxy groups can be successfully synthesized in three-step process: addition, polyesterification, and oxidation process. The novel materials can be applied as a potential polar matrices for preparation of wood flour filled copolymers due to their "compatibility" with hydrophilic wood flour surface which resulted with stronger interactions between wood and polymer surface and thus producing copolymers which were characterized by improved thermal and mechanical properties compared to unfilled copolymers which was confirmed based on DSC, TGA, DMA analyses, and mechanical test: three-point bending test and Brinell's hardness.

Open Access This article is distributed under the terms of the Creative Commons Attribution Noncommercial License which permits any noncommercial use, distribution, and reproduction in any medium, provided the original author(s) and source are credited.

\section{References}

1. Wang T, Wan PY, Yu QP, Yu M (2008) Synthesis and characterization of dicyclopentadiene-cresol epoxy resin. Polym Bull 59:787-793 
2. Hsiue GH, Shiao SJ, Wei HF, Kuo WJ, Shai YA (2001) Novel phosphorus-containing dicyclopentadiene-modified phenolic resins for flame retardancy applications. J Appl Polym Sci 79:342-349

3. Johnson KG, Yang LS (2003) Preparation, properties and applications of unsaturated polyesters: modern polyesters chemistry and technology of polyesters and copolyesters. Wiley, Chichester

4. Crivello JV, Soyoung S (2000) Synthesis and cationic photopolymerization of novel monomers based on dicyclopentadiene. Chem Mater 12:3674-3680

5. Bruson HA, Reiner TW (1945) The chemistry of DCPD. I. Hydratation and rearrangement. J Am Chem Soc 67:723-728

6. Zhang X, Zhang Z, Xia X, Zhang Z, Xu W, Xiong Y (2007) Synthesis and characterization of a novel cycloaliphatic epoxy resin starting from dicyclopentadiene. Eur Polym J 43:2149-2154

7. Bergmann F, Japhe H (1947) On the addition of formic acid to dicyclopentadiene. J Am Chem Soc 69:1826-1827

8. McGary CW, Patrick CT, Smith PL (1963) Resins from endo-dicyclopentadiene dioxide. J Appl Polym Sci 7:1-14

9. Lubin G (1982) Handbook of composites. Van Nostrand Reinhold, New York

10. Yang TCK, Tsai SHY, Wang SF, Ch Juan (2002) Dielectric and thermal studies of inorganic microfillers on polymer microwave substrates-metallocene cyclic olefin copolymers (COC). Compos Sci Technol 62:655-661

11. Lee SM (1988) Epoxy resins. Mercel Dekker, New York

12. Xu K, Chen M, Zhang K, Hu J (2004) Synthesis and characterization of novel epoxy resin bearing naphthyl and limonene moieties and its cured polymer. Polymer 45:1133-1140

13. Park SJ, Jin FL (2004) Thermal stabilities and dynamic mechanical properties of sulfone-containing epoxy resin cured with anhydride. Polym Degrad Stab 186:517-520

14. Xie M, Wang Z, Zhao Y (2001) Synthesis and properties of a novel, liquid, trifunctional cycloaliphatic epoxide. J Polym Sci A Polym Chem 39:2799-2804

15. Wang Z, Xie M, Zhao Y, Yu Y, Fang S (2003) Synthesis and properties of novel liquid ester-free reworkable cycloaliphatic diepoxides for electronic packing applications. Polymer 44:923-939

16. Nguyen T, Zavarin E, Barral EM II (1981) Thermal analysis of lignocellulose materials. Part II. Modified materials. J Macromol Sci Rev Macromol Chem 21:1-60

17. Hon David NS, Shiraishi N (1991) Wood and cellulose chemistry. Mercel Dekker Inc, New York and Basel

18. Ray D, Sarkar BK, Bose NR (2002) The impact fatigue behaviour of vinylester resin matrix composites reinforced with alkali-treated jute fibres. Composites A 33:233-241

19. Rana AK, Mitra BC, Banerjee AN (1999) Short jute fibre-reinforced polypropylene composites: dynamic mechanical study. J Appl Polym Sci 71:531-539

20. Saha AK, Das S, Bhatta D, Mitra BC (1999) Study of jute fibre reinforced polyester composites by dynamic mechanical analysis. J Appl Polym Sci 71:1505-1513

21. Akita K, Kase MJ (1967) Determination of kinetics parameters for pyrolisis of cellulose and cellulose treated with ammonium phosphate by differential thermal analysis and thermal gravimetric analysis. J Polym Sci A Polym Chem 5:833-848

22. Alfthan E, de Alf Ruvo, Brown W (1973) Glass transition temperature of oligosaccharides. Polymer 4:329-330

23. Ray D, Sarkar BK, Das S, Rana AK (2002) Dynamic mechanical and thermal analysis of vinylesterresin-matrix composites reinforced with untreated and alkali treated jute fibres. Compos Sci Technol 62:911-917

24. Worzakowska M (2009) Chemical modification of unsaturated polyesters. Influence of polyester's structure on thermal and viscoelastic properties of low styrene content copolymers. J Appl Polym Sci 114:720-731

25. Chu NJ (1970) The conformation of the anhydrocellobiose units in cellulose I and II. J Appl Polym Sci $14: 3129-3136$

26. Beall FC (1986) Thermal degradation of wood. In: Bever MB (ed) Encyclopedia of materials science and engineering, 1st edn. Pergamon Press, Oxford

27. Tang MM, Bacon R (1964) Carbonization of cellulose fibres-I. Low temperature pyrolysis. Carbon 2:211-214 